\title{
Clinical Factors Predicting Better Survival Outcome for Pulmonary Metastasectomy of Hepatocellular Carcinoma
}

\author{
Tse-Ming Kuo $^{a} \quad K^{2}$ ai-Ming Chang ${ }^{b}$ Tsun-I Cheng ${ }^{a} \quad K^{\prime}$ Kuo-Jang Kao ${ }^{b}$ \\ a Division of Gastroenterology and Hepatobiliary Disease, Department of Medicine, and \\ ${ }^{b}$ Department of Research, Koo Foundation Sun-Yat Sen Cancer Center, Taipei, Taiwan, ROC
}

\section{Keywords}

Extrahepatic metastasis · Hepatocellular carcinoma - Outcome · Prognosis prediction .

Surgery

\begin{abstract}
Background: In patients with lung metastasis of hepatocellular carcinoma (HCC), it remains uncertain how a better survival outcome can be predicted after metastasectomy. This study aims to identify clinical factors that may be used to guide patient selection for such a therapeutic modality. A total of 28 patients who received pulmonary metastasectomy for HCC between 1993 and 2012 were identified. All relevant clinical factors were extracted from medical records up to September 2015. Patients were classified into high- and low-risk groups according to survival outcome after metastasectomy. All pertinent clinical factors were analyzed for correlation with survival outcome. Summary: The overall survival of 28 patients after pulmonary metastasectomy was studied first. The survival curve was biphasic and reached a plateau at 40 months after metastasectomy. The results indicate the presence of 2 groups of patients with a different survival outcome. Among all clinical parameters, remission status in the liver before pulmonary metastasectomy and distant metastasis-free interval between the last treatment of HCC and the occurrence of lung metastasis were found to be significantly associated with excellent survival outcome after pulmonary metastasectomy ( $p=0.019$ and 0.007 by Fisher exact test, and $p=0.002$ and 0.0002 by Cox regression analysis).
\end{abstract}

(C) 2017 S. Karger AG, Basel

\section{Introduction}

Hepatocellular carcinoma (HCC) is the fifth most common cancer in men and the ninth most common in women worldwide. Globally, it is the second leading cause of cancer death in men and the sixth leading cause among women [1]. For the treatment of HCC, there are 
Kuo et al.: Clinical Factors Predicting Better Survival Outcome for Pulmonary

Metastasectomy of Hepatocellular Carcinoma

many modalities, including curative hepatic surgery, liver transplantation, transcatheter hepatic arterial chemoembolization, local ablation by radiofrequency or percutaneous ethanol injection, radiation therapy, and targeted therapy with sorafenib. The use of these treatment modalities has improved the survival of the disease to different extents.

HCC is known for a high frequency of intrahepatic recurrence and distant metastasis. Extrahepatic metastasis is usually indicative of impending demise of patients. According to a study by Chan et al. [2], the median length of patient survival was 6.9 months (range, 0.8-96 months) and the overall 1-, 3-, and 5-year survival rates were 31,7 , and $4 \%$, respectively, in patients with extrahepatic metastases. The most frequent sites of extrahepatic metastases were the lungs (47\%) followed by lymph nodes (45\%), bone (37\%), and adrenal glands (12\%) [3]. Once pulmonary metastasis was found, the average survival was 3.3 months [4]. Systemic chemotherapy of HCC provided marginal benefit with frequent toxic side effects [5].

Although the lung is the most frequent initial site of metastasis and pulmonary metastasectomy was reported to improve survival in small numbers of highly selected patients with metastasis restricted to the lungs without residual or recurrent tumor in the liver [6-8], it remains uncertain how patients with pulmonary metastasis should be selected for metastasectomy. The original surgical criteria for metastatic tumors in the lungs were proposed by Thomford et al. [9] in 1965. Although HCC was not included in the study of Thomford et al. [9], these criteria were adopted for pulmonary metastasectomy in the studies reported later for HCC [10-14]. These criteria were that the primary tumor was judged to be under control, radiological evidence of metastasis was limited to one lung, and there was no evidence of metastatic disease elsewhere in the body. The criteria proposed by Thomford and colleagues were slightly modified later by McCormack [15] in 1990. The revised criteria were that a patient was a suitable candidate for surgery, the primary tumor was controlled or controllable, no extrathoracic metastasis existed, no better treatment method was available, and complete resection was possible. If there was recurrence in the liver, the recurrent disease was controlled first to prevent further metastatic seeding.

Nevertheless, many patients who had met these criteria did not do well after metastasectomy. It is therefore important to identify reliable clinical parameters that can be used for better selection of patients to receive pulmonary metastasectomy. To achieve this goal, we identified 28 patients who received pulmonary metastasectomy at our institution between January 1993 and March 2012. Multiple pertinent clinical factors were extracted and investigated for association with survival outcome after pulmonary metastasectomy in these patients. The results are reported herein.

\section{Materials and Methods}

The ICD9 code was used to identify all patients with HCC who developed lung metastasis and received pulmonary metastasectomy between 1993 and 2012. Out of a total of 2,817 HCC patients treated at our center, $347(12.3 \%)$ had developed lung metastasis. Only $28(1.0 \%)$ patients with lung metastasis received pulmonary metastasectomy. These 28 patients were included in the present study.

All HCC patients treated at our cancer center were regularly followed up every 3 months after treatment. Physical examination, chest radiography, serum alpha-fetoprotein (AFP), liver function test, and ultrasound of the upper abdomen were performed during each follow-up visit. Additional abdominal computed tomography (CT) or magnetic resonance imaging was ordered, if sonography of the upper abdomen could not provide a conclusive answer. All patients received at least 1 annual CT or magnetic resonance imaging of the upper abdomen. Bone scan and radiography were ordered if clinically indicated.

When a pulmonary nodule was discovered by chest X-ray and/or CT scan during a follow-up visit, CT or sonography-guided needle biopsy was offered to patients for diagnosis of metastasis. Patients who were proven to have HCC metastasis in the lungs by biopsy or strongly suspected by imaging studies were referred to a pulmonary surgeon for evaluation of possible resection. The criteria for metastasectomy included (1) 
Kuo et al.: Clinical Factors Predicting Better Survival Outcome for Pulmonary

Metastasectomy of Hepatocellular Carcinoma

Table 1. Definitions of key clinical parameters used in the study

\begin{tabular}{ll} 
Complete remission & $\begin{array}{l}\text { Clinical status that is free of any residual primary liver tumor, intrahepatic } \\
\text { recurrence, and extrahepatic nonpulmonary metastasis right before pulmonary } \\
\text { metastasectomy }\end{array}$ \\
\hline Partial response & $\begin{array}{l}\text { Clinical status not meeting the criteria of complete remission and showing a } \\
\text { stable or reduced tumor size in the liver }\end{array}$ \\
\hline $\begin{array}{l}\text { Distant metastasis-free } \\
\text { interval }\end{array}$ & $\begin{array}{l}\text { Interval between the date of the last treatment of HCC in the liver and the date } \\
\text { of the first occurrence of lung metastasis; no metastatic disease elsewhere } \\
\text { concurrently }\end{array}$ \\
\hline Disease-free interval & $\begin{array}{l}\text { Interval between the date of curative surgery of intrahepatic lesion and the date } \\
\text { of the first occurrence of lung metastasis }\end{array}$ \\
\hline Overall survival & $\begin{array}{l}\text { Time from the initial pulmonary metastasectomy to the last follow-up date or } \\
\text { death }\end{array}$
\end{tabular}

possibility for complete resection, (2) no evidence of uncontrolled intrahepatic and extrapulmonary lesions, and (3) suitable physical condition for surgery. For our study, we identified 28 patients who received surgical resection of metastatic HCC in the lungs between January 1993 and March 2012. Nine of the 28 patients received preoperative fine needle biopsy. In the remaining 19 patients, preoperative fine needle biopsy was not performed, and diagnoses of metastatic HCC in the lungs were later confirmed histologically on the resected surgical specimens. The pertinent clinical factors selected for our study included age, gender, type of viral hepatitis (B vs. C), initial liver tumor T stage (I/II vs. III/IV), resection margin (negative vs. positive), response after initial treatment of primary HCC in the liver (complete remission vs. partial response), distant metastasis-free interval between the last treatment of HCC in the liver and occurrence of lung metastasis, number of metastatic nodules in the lungs, unilateral or bilateral involvement of pulmonary metastasis, size of the metastatic tumor, type of pulmonary resection (lobectomy vs. wedge resection), surgical method (thoracotomy vs. video-assisted thoracic surgery), and number of metastasectomy procedures. The causes of death were also determined. Definitions of key clinical parameters used in the study are summarized in Table 1. Open thoracotomy was performed on all patients who were surgically treated before $2005(n=16)$. After 2005, video-assisted thoracic surgery $(n=12)$ was performed on all patients.

For statistical analysis, the 28 patients were divided into high- and low-risk groups based on the length of their survival after pulmonary metastasectomy. Associations of all clinical parameters with survival outcome were studied using the Fisher exact test and Cox regression analysis. Permutation $t$ test was used in the comparison between the 2 groups to avoid the assumption of normality.

\section{Results}

\section{Patient Characteristics and Risk Classification}

Clinical characteristics of the 28 patients included in the study are summarized in Table 2. To identify patients with high or low risk for disease progression after pulmonary metastasectomy, we first studied the overall survival after pulmonary metastasectomy. As shown in Figure 1, the survival curve appears biphasic and reaches a plateau at approximately 40 months after pulmonary metastasectomy. The result of this study indicates that there were 2 groups of patients. One group had a shorter survival than the other. We therefore used a survival of 40 months as the cutoff to define patients as high or low risk for disease progression after pulmonary metastasectomy. There were 15 patients in the high-risk group and 12 patients in the low-risk group. One surviving patient, who had a follow-up of $<40$ months, was regarded as indeterminate for risk of disease progression and not included in the comparative analysis. 


\section{Liver \\ Cancer}

Table 2. Clinical characteristics of the 28 patients included in the study

\begin{tabular}{l|l}
\hline \multicolumn{2}{l|}{ Liver Cancer 2017;6:297-306 } \\
\hline DOI: 10.1159/000477134 & $\begin{array}{l}\text { ( ) 2017 S. Karger AG, Basel } \\
\text { www.karger.com/lic }\end{array}$ \\
\hline
\end{tabular}

Kuo et al.: Clinical Factors Predicting Better Survival Outcome for Pulmonary Metastasectomy of Hepatocellular Carcinoma
Gender, $n(\%)$
Male
Female

Age, years

Mean \pm SD

Range

Histology differentiation grade, $n(\%)$

Well

Moderate

Poor

T stage $\mathrm{e}^{\mathrm{a}}, n(\%)$

II

IIIA

IIIB

IV

M stage ${ }^{\mathrm{a}}, n(\%)$

0

History of viral hepatitis ${ }^{\mathrm{b}}, n(\%)$

Hepatitis B

Hepatitis C

Indocyanine green clearance test at $15 \mathrm{~min}, \%$ Median

Mean \pm SD

Maximum size of liver tumor, $\mathrm{cm}$

Median

Mean \pm SD

Number of tumors in the liver

Median

Mean \pm SD

Number of metastatic tumors in the lungs Median

Mean \pm SD

Laterality of pulmonary metastasis, $n(\%)$ Unilateral

Bilateral

Size of metastatic tumors, $\mathrm{cm}$

Median

Mean \pm SD

Disease-free interval, months

Median

Mean \pm SD

Distant metastasis-free interval, months

Median

Mean \pm SD

Type of pulmonary resection, $n(\%)$

Wedge resection

Lobectomy and others

Surgical method, $n(\%)$

Open thoracotomy

Video-assisted thoracic surgery
$19(68)$

$9(32)$

$49.4 \pm 15.2$

$19-71$

$3(12)$

$17(65)$

$6(23)$

2 (7)

3 (11)

$17(61)$

5 (18)

$1(3)$

25 (89)

$23(82)$

2 (7)

9

$11.9 \pm 8.6$

9

$9.2 \pm 4.5$

1.5

$2.2 \pm 2.2$

1.5

$2.0 \pm 1.3$

20 (71)

8 (29)

2

$2.6 \pm 1.8$

22.7

$22.1 \pm 17.5$

3.5

$11.1 \pm 15.6$

16 (57)

12 (43) a $\mathrm{T}$ and $\mathrm{M}$ stages were determined according to AJCC 7 th edition. $\mathrm{T}$ and $\mathrm{M}$ stages were the initial tumor stages when HCC was diagnosed.

b The other 5 patients were negative for hepatitis $\mathrm{B}$ and $\mathrm{C}$ infection serologically. 


\section{Liver Cancer}

Fig. 1. Probability of survival of all 28 patients after initial pulmonary metastasectomy. The 1-, 2-, and 5-year survival probabilities were $85.7,67.9$, and $46.2 \%$, respectively. The survival curve appeared to reach a plateau at 40 months after metastasectomy which was used to define patients with low and high risk of disease progression. These 2 groups of patients were compared to identify parameters that could be used to select patients who would benefit most from metastasectomy.

Fig. 2. The percentage of patients who remained metastasis-free was plotted against months after the last treatment of HCC. This plot appears biphasic with an inflection point (dashed line) at approximately 6 months. Six months was therefore chosen as the cutoff for the parameter of distant metastasis-free interval that was used for statistical comparison in Table 3.

\begin{tabular}{l|l}
\hline Liver Cancer 2017;6:297-306 \\
\hline DOI: 10.1159/000477134 & $\begin{array}{l}\text { @ 2017 S. Karger AG, Basel } \\
\text { www.karger.com/lic }\end{array}$ \\
\hline
\end{tabular}

Kuo et al.: Clinical Factors Predicting Better Survival Outcome for Pulmonary Metastasectomy of Hepatocellular Carcinoma
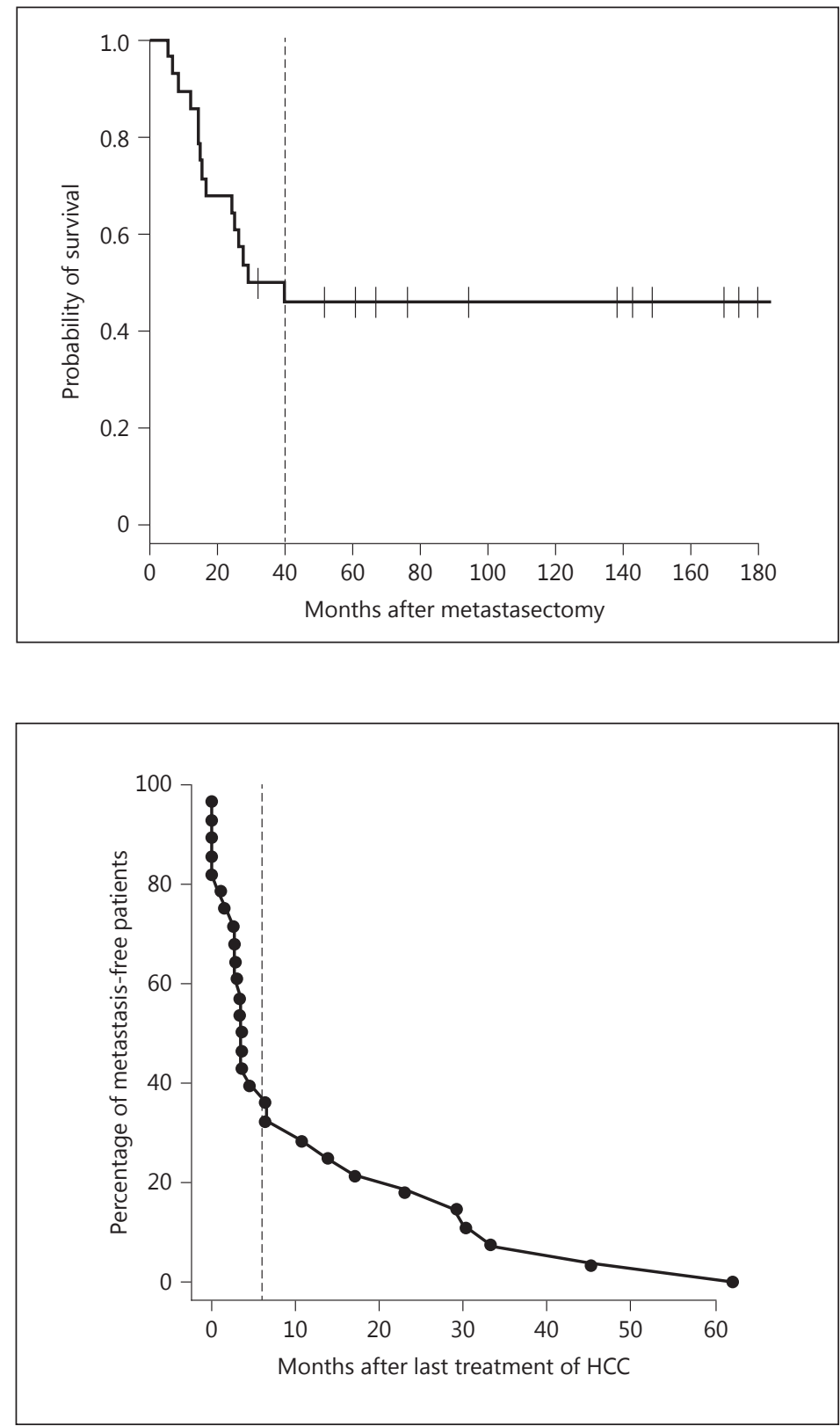

\section{Comparison between High- and Low-Risk Patients}

Clinical parameters listed in Table 3 were compared between patients with high and those with low risk for disease progression after metastasectomy using the Fisher exact test and Cox regression. We found that the remission status in the liver before pulmonary metastasectomy was a major factor significantly associated with overall survival after metastasectomy (Fisher exact test, $p=0.019$; Cox regression, $p=0.002$ ). A distant metastasis-free interval of $\geq 6$ months before metastasectomy was also associated with better overall survival of $>40$ months (Fisher exact test, $p=0.007$; Cox regression, $p=0.0002$ ). We chose 6 months to define the distant metastasis-free interval for our study, because the percentage of patients who remained distant metastasis-free after the last liver HCC treatment as a function of time appeared to be biphasic with an inflection point at approximately 6 months (Fig. 2). 
Kuo et al.: Clinical Factors Predicting Better Survival Outcome for Pulmonary
Metastasectomy of Hepatocellular Carcinoma

Table 3. Correlation of clinical variables with low and high risk for disease progression and poor survival after pulmonary metastasectomy

\begin{tabular}{|c|c|c|c|c|c|}
\hline & \multirow{2}{*}{$\begin{array}{l}\text { Low-risk } \\
\text { group, } \\
n(\%)\end{array}$} & \multirow{2}{*}{$\begin{array}{l}\text { High-risk } \\
\text { group, } \\
n(\%)\end{array}$} & \multicolumn{2}{|l|}{$p$ value } & \multirow{2}{*}{$\begin{array}{l}\text { Hazard ratio } \\
(95 \% \mathrm{CI})\end{array}$} \\
\hline & & & $\begin{array}{l}\text { Fisher } \\
\text { exact test }\end{array}$ & $\begin{array}{l}\text { Cox } \\
\text { regression }\end{array}$ & \\
\hline Age at initial treatment of HCC & & & 0.704 & 0.966 & $1.00^{\mathrm{a}}(0.96,1.04)$ \\
\hline$<50$ years & $7(50)$ & $7(50)$ & & & \\
\hline$\leq 50$ years & $5(38)$ & $8(62)$ & & & \\
\hline Gender & & & 0.696 & 0.836 & $0.89(0.30,2.61)$ \\
\hline Female & $3(38)$ & $5(63)$ & & & \\
\hline Male & $9(47)$ & $10(53)$ & & & \\
\hline Hepatitis $\mathrm{B}^{\mathrm{b}}$ & & & 0.342 & 0.124 & $0.37(0.12,1.19)$ \\
\hline Negative & $1(20)$ & $4(80)$ & & & \\
\hline Positive & $11(50)$ & $11(50)$ & & & \\
\hline Tumor T stage & & & 1.000 & 0.596 & $1.47(0.33,6.51)$ \\
\hline $\mathrm{I} / \mathrm{II}$ & $2(50)$ & $2(50)$ & & & \\
\hline III/IV & $10(43)$ & $13(57)$ & & & \\
\hline Resection margin & & & 0.590 & 0.184 & $2.67(0.71,10.05)$ \\
\hline Negative & $11(55)$ & $9(45)$ & & & \\
\hline Positive & $1(25)$ & $3(75)$ & & & \\
\hline Status of primary liver tumor & & & 0.019 & 0.002 & $5.44(1.91,15.51)$ \\
\hline $\mathrm{CR}$ & $11(61)$ & $7(39)$ & & & \\
\hline PR/recurrence & $1(11)$ & $8(89)$ & & & \\
\hline Disease-free interval & & & 0.107 & 0.051 & $0.9662(0.93,1.00)$ \\
\hline$<12$ months & $2(20)$ & $8(80)$ & & & \\
\hline$\geq 12$ months & $10(61)$ & $7(39)$ & & & \\
\hline Distant metastasis-free interval & & & 0.007 & 0.0002 & $0.85^{\mathrm{a}}(0.72,0.99)$ \\
\hline$<6$ months & $4(24)$ & $13(76)$ & & & \\
\hline$\geq 6$ months & $8(80)$ & $2(20)$ & & & \\
\hline Number of metastatic nodules & & & 0.252 & 0.945 & $0.99^{\mathrm{a}}(0.69,1.41)$ \\
\hline 1 & $8(57)$ & $6(43)$ & & & \\
\hline$\geq 2$ & $4(31)$ & $9(69)$ & & & \\
\hline Laterality & & & 0.091 & 0.121 & $2.35(0.83,6.62)$ \\
\hline Unilateral & $11(55)$ & $9(45)$ & & & \\
\hline Bilateral & $1(14)$ & $6(86)$ & & & \\
\hline Maximum size of the metastatic nodules & & & 0.662 & 0.716 & $0.95^{\mathrm{a}}(0.71,1.26)$ \\
\hline$<3.0 \mathrm{~cm}$ & $10(48)$ & $11(52)$ & & & \\
\hline$\geq 3.0 \mathrm{~cm}$ & $2(33)$ & $4(67)$ & & & \\
\hline Type of pulmonary resection & & & 0.569 & 0.562 & $1.74(0.23,13.26)$ \\
\hline Lobectomy & $2(67)$ & $1(33)$ & & & \\
\hline Wedge & $10(42)$ & $14(58)$ & & & \\
\hline Surgical method & & & 0.707 & 0.878 & $0.92(0.33,2.59)$ \\
\hline Thoracotomy & $6(40)$ & $9(60)$ & & & \\
\hline Video-assisted thoracic surgery & $6(50)$ & $6(50)$ & & & \\
\hline Number of pulmonary surgeries & & & 1.000 & 0.338 & $0.80^{\mathrm{a}}(0.48,1.34)$ \\
\hline 1 & $9(45)$ & $11(55)$ & & & \\
\hline$\geq 2$ & $3(43)$ & $4(57)$ & & & \\
\hline
\end{tabular}

CR, complete remission; PR/recurrence, partial response or recurrence in liver. ${ }^{a}$ Age, disease-free interval, distant metastasis-free-interval, number of metastatic nodules, maximum size of the metastatic nodule, and number of pulmonary surgeries were considered as continuous variables in Cox regression for hazard ratio calculation. With a continuous variable, hazard ratio indicates change in risk due to each unit rise. ${ }^{\mathrm{b}}$ One hepatitis B-positive patient was excluded due to indeterminate serological status. 
Kuo et al.: Clinical Factors Predicting Better Survival Outcome for Pulmonary

Metastasectomy of Hepatocellular Carcinoma

Table 4. Tumor recurrence status and cause of death of the 15 patients who died during follow-up

\begin{tabular}{lll}
\hline Patient & Final status of tumor recurrence & Cause of death \\
\hline 1 & HCC recurrence in the liver & Liver tumor progression \\
2 & HCC recurrence in the liver and recurrent lung metastasis & Unknown \\
3 & HCC recurrence in the liver & Liver tumor progression \\
4 & HCC recurrence in the liver, recurrent lung metastasis and brain metastasis & Brain metastasis \\
5 & Lost to follow-up & Unknown \\
\hline 6 & HCC recurrence in the liver, recurrent lung metastasis and brain metastasis & Brain metastasis \\
7 & HCC recurrence in the liver & Liver tumor progression \\
8 & HCC recurrence in the liver and recurrent lung metastasis & Liver tumor progression \\
9 & HCC recurrence in the liver and recurrent lung metastasis & Liver tumor progression and \\
& & respiratory failure \\
10 & HCC recurrence in the liver & Liver tumor progression \\
\hline 11 & HCC recurrence in the liver and brain metastasis & Brain metastasis \\
12 & HCC recurrence in the liver and recurrent lung metastasis & Liver tumor progression \\
13 & HCC recurrence in the liver and recurrent lung metastasis & Liver tumor progression \\
14 & Lost to follow-up & Unknown \\
15 & HCC recurrence in the liver & Bleeding of esophageal varices \\
\hline
\end{tabular}

\section{Causes of Death}

Of the 28 patients included in this study, 15 patients died. All 15 deceased patients belonged to the high-risk group. The causes of their death and final status of tumor recurrence were investigated and are summarized in Table 4. Three patients were lost to follow-up and died according to the public record. The causes of their death were unavailable. Of the remaining 12 patients, all had recurrent HCC in the liver with disease progression. These patients were treated with TACE, hepatectomy, radiofrequency ablation, percutaneous ethanol injection, and/or radiation as needed according to their clinical conditions. Eight of the 12 patients died of liver failure due to liver tumor progression. Seven of the deceased patients developed recurrent lung metastases. Nevertheless, only 1 patient died of respiratory failure associated with recurrent lung metastasis in the presence of coexisting liver tumor progression. Three patients died of brain metastasis. One patient died of bleeding esophageal varices. Development of intrahepatic recurrence with hepatic failure and brain metastasis were the major causes of death. The finding of low likelihood of dying from respiratory failure suggests that recurrent lung metastasis was not a major cause of death among patients treated with pulmonary metastasectomy.

Three of the 7 patients with recurrent lung metastasis received repeated pulmonary metastasectomy. One had pulmonary metastasectomy a total of 3 times and died of brain metastasis 38 months after the first metastasectomy. The other 2 patients had pulmonary metastasectomy twice in total. Both died of liver tumor progression and one of them also developed respiratory failure due to lung metastasis. The other 4 patients with recurrent lung metastasis did not receive metastasectomy due to liver tumor progression.

\section{Discussion}

In order to identify clinical factors that may be used to identify HCC patients who are suitable for pulmonary metastasectomy, we identified 28 patients who received such treatment at our institution from 1993 to 2012. Like many other studies in the past [6-8, $10-14,16-25]$, only a limited number of patients was available for the study. To effectively 
Kuo et al.: Clinical Factors Predicting Better Survival Outcome for Pulmonary Metastasectomy of Hepatocellular Carcinoma

use the limited number of patients, we conducted a survival analysis of these patients at the beginning and found a biphasic survival curve indicating that there were 2 groups of patients (Fig. 1). One group had limited survival and the other had excellent long-term survival after pulmonary metastasectomy. The presence of these 2 groups of patients offered us an opportunity to conduct a comparative study addressing our question.

Through comparison of these 2 groups of patients, we found 2 clinical factors that were associated with long-term survival outcome after pulmonary metastasectomy, namely remission status of HCC in the liver (complete remission vs. partial remission) at the time of metastasectomy, and metastasis-free interval of $>6$ months between the last treatment of HCC in the liver and the occurrence of pulmonary metastasis. Patients who met these 2 criteria survived longer than 40 months after metastasectomy. The results indicate that these 2 clinical parameters may be useful for identifying patients who should receive pulmonary metastasectomy to gain long-term survival outcome. However, our study was unable to answer the question of whether the high-risk patients who died within 40 months after initial metastasectomy benefited from metastasectomy or not. Thus, the findings of our study cannot be used to exclude patients who did not meet the low-risk criteria for pulmonary metastasectomy.

According to earlier and recently published studies, disease-free interval (DFI) [11, 16, $18,26]$, AFP level [16-19], history of recurrence [20], and $\leq 2$ pulmonary metastatic tumor nodules [11] are prognostic for patients undergoing pulmonary metastasectomy. The results of our study support that DFI and status of primary liver tumor are indeed important prognostic factors. The parameter of distant metastasis-free interval used in our study is part of the reported DFI. Distant metastasis-free interval was used in our study because not all our patients received hepatic resections as the initial treatment. Eighteen of our patients received hepatic resection first and 9 of them also received additional treatment of TACE and/or radiofrequency ablation. Seven other patients were treated with TACE initially and received hepatic resection later. The other 3 patients did not receive any hepatic resection and were treated with repeated TACE, alcohol injection, and/or radiofrequency ablation. Consequently, durations of treatment of HCC in the liver varied significantly among our patients. The median duration between the first treatment and the last treatment of the liver was 6.6 months with a mean and standard deviation of 11.5 and 13.6 months. We therefore adopted "distant metastasis-free interval" to avoid significantly varying durations devoted to treat HCC in the liver. We believe that the distant metastasis-free interval could more directly reflect the biology of tumor growth for lung metastasis, because it assesses the time period between completion of liver tumor treatment and the clinical appearance of lung metastasis. We investigated the use of DFI of 12 months as a prognostic parameter (Table 3). DFI was defined as the duration between the initial treatment of HCC in the liver and the detection of pulmonary metastasis. DFI of 12 months did not show a significant association and only showed a trend with survival outcome after metastasectomy (Table 3). The lack of significant association was likely due to the high degree of variation in the duration devoted to control HCC in the liver as mentioned above. We also used status of primary liver tumor before pulmonary metastasectomy as a variable in our study. "Status of primary liver tumor" includes complete remission (no recurrence) and partial remission of liver tumor or recurrence in the liver after initial treatment which is similar to "history of recurrence" [20].

On the one hand, the results of our study are consistent with the findings of previous studies, because the distant metastasis-free interval used by us was part of DFI used in the reported studies. On the other hand, there was a major difference between our study and a previously reported study [26]. This difference was inclusion of many patients treated with different therapeutic modalities other than hepatic resection to control HCC in the liver before metastasectomy. The results of our study indicate that patients not eligible for hepatic 
resection initially may benefit from pulmonary metastasectomy and gain a better survival outcome as long as their HCC in the liver can be controlled and lung metastasis does not occur within 6 months after the last treatment of HCC in the liver.

Regarding AFP as a prognostic and predictive factor, we did not include this variable in our analyses, because AFP levels were not consistently determined shortly before pulmonary metastasectomy. The time interval between AFP measurements and the surgical procedure varied significantly from 3 to 107 days.

In our study, we included patients who had metastasectomy up to March 2012. The national health insurance coverage of sorafenib treatment was not available until September 2012. In September 2012, 14 patients were alive. Among these 14 patients, 1 died of liver tumor progression and brain metastasis in September 2012. Another patient died of bleeding esophageal varices in November 2012. This patient did not have recurrent pulmonary metastasis but had intrahepatic recurrence and tumor progression. Twelve of these patients were still alive up to September 2015. No patient was treated with sorafenib or chemotherapy. There were no lung metastasis patients without surgery who were treated with sorafenib for comparison. Therefore, we were unable to compare the difference of survival between such two groups of patients.

Although our study spanned nearly 20 years from 1993 to 2011, 27 out of 28 patients were diagnosed and treated after 1999. Only 1 patient included in the study was diagnosed and treated before 1999 (in 1993). Thus, the long-spanning duration of our study should not have a major impact on our findings. We also recognize the limited number of patients in our study. Nevertheless, findings generated from the present study are consistent with recently reported studies [25-26]. Our findings and those reported by Mizuguchi et al. [25] and Takahashi et al. [26] could be clinically useful to identify patients who may benefit most from metastasectomy for lung metastasis of HCC.

\section{Statement of Ethics}

This study was approved by the Institution Review Board (ID number 20160222A) of Koo Foundation Sun Yat-Sen Cancer Center and the use of informed consent was exempted for the use of de-identified data. This study was conducted in accordance with the ethical standards of the 1964 Declaration of Helsinki and its later amendments.

\section{Disclosure Statement}

The authors declare no conflicts of interest.

\section{References}

1 Torre LA, Bray F, Siegel RL, Ferlay J, Lortet-Tieulent J, Jemal A: Global cancer statistics, 2012. CA Cancer J Clin 2015;65:87-108.

2 Chan KM, Yu MC, Wu TJ, Lee CF, Chen TC, Lee WC, Chen MF: Efficacy of surgical resection in management of isolated extrahepatic metastases of hepatocellular carcinoma. World J Gastroenterol 2009;15:5481-5488.

3 Uka K, Aikata H, Takaki S, Shirakawa H, Jeong SC, Yamashina K, Hiramatsu A, Kodama H, Takahashi S, Chayama K: Clinical features and prognosis of patients with extrahepatic metastases from hepatocellular carcinoma. World J Gastroenterol 2007;13:414-420.

4 Liou WY, Hung JY, Chen JW, Hou JJ, Hsu TH, Wang TH, Chong IW, Hwang JJ, Huang MS: Pulmonary metastasis of hepatocellular carcinoma. Thorac Med 2001;16:95-101.

5 Forner A, Llovet J, Bruix J: Hepatocellular carcinoma. Lancet 2012;379:1245-1255.

6 Lam CM, Lo CM, Yuen WK, Liu CL, Fan ST: Prolonged survival in selected patients following surgical resection for pulmonary metastasis from hepatocellular carcinoma. Br J Surg 1998;85:1198-1200. 
Kuo et al.: Clinical Factors Predicting Better Survival Outcome for Pulmonary Metastasectomy of Hepatocellular Carcinoma

7 Chen YJ, Hsu HS, Hsieh CC, Wu YC, Wang LS, Hsu WH, Huang MH, Huang BS: Pulmonary metastasectomy for hepatocellular carcinoma. J Chin Med Assoc 2004;67:621-624.

8 Gwak GY, Jung JO, Sung SW, Lee HS: Long-term survival after pulmonary metastasectomy of hepatocellular carcinoma; treatment outcome or nature history? Hepatogastroenterology 2004;51:1428-1433.

9 Thomford NR, Woolner LB, and Clagett OT, Minn R: The surgical treatment of metastatic tumors in the lungs. J Thorac Cardiovasc Surg 1965;49:357-363.

10 Tomimaru Y, Sasaki Y, Yamada T, Eguchi H, Takami K, Ohigashi H, Higashiyama M, Ishikawa O, Kodama K, Imaoka S: The significance of surgical resection for pulmonary metastasis from hepatocellular carcinoma. Am J Surg 2006;192:46-51.

11 Lee CY, Bae MK, Park IK, Kim DJ, Lee JG, Choi JS, Han KH, Chung KY: Surgical resection for pulmonary metastasis from hepatocellular carcinoma: analysis of prognosis in relation to primary control. J Surg Oncol 2010; 101:239-243.

12 Kwon JB, Park K, Kim YD, Seo JH, Moon SW, Cho DG, Kim YW, Kim DG, Yoon SK, Lim HW: Clinical outcome after pulmonary metastasectomy from primary hepatocellular carcinoma: analysis of prognostic factors. World J Gastroenterol 2008;14:5717-5722.

13 Nakajima J, Tanaka M, Matsumoto J, Takeuchi E, Fukami T, Takamoto S: Appraisal of surgical treatment for pulmonary metastasis from hepatocellular carcinoma. World J Surg 2005;29:715-718.

14 Kawamura M, Nakajima J, Matsuguma H, Horio H, Miyoshi S, Nakagawa K, Fujisawa T, Kobayashi K; The Metastatic Lung Tumor Study Group of Japan: Surgical outcomes for pulmonary metastases from hepatocellular carcinoma. Eur J Cardiothorac Surg 2008;34:196-199.

15 McCormack P: Surgical resection of pulmonary metastases. Semin Surg Oncol 1990;6:297-302.

16 Nakagawa T, Kamiyama T, Nakanishi K, Yokoo H, Kamachi H, Matsushita M, Todo S: Pulmonary resection for metastases from hepatocellular carcinoma: factors influencing prognosis. J Thorac Cardiovasc Surg 2006;131: 1248-1254.

17 Ohba T, Yano T, Yoshida T, Kawano D, Tsukamoto S, Shoji F, Taketomi A, Saitsu H, Takeo S, Maehara Y: Results of surgical resection of pulmonary metastasis from hepatocellular carcinoma: prognostic impact of the preoperative serum alpha-fetoprotein level. Surg Today 2012;42:526-531.

18 Kuo SW, Chang YL, Huang PM, Hsu HH, Chen JS, Lee JM, Lee PH, Lee YC: Prognostic factors for pulmonary metastasectomy in hepatocellular carcinoma. Ann Surg Oncol 2007;14:992-997.

19 Cho S, Ryu KM, Hwang YJ, Lee EB: Prognostic factors for pulmonary metastasectomy in the treatment of hepatocellular carcinoma. J Thorac Oncol 2010;5:1251-1254.

20 Kitano K, Murayama T, Sakamoto M, Nagayama K, Ueno K, Murakawa T, Nakajima J: Outcome and survival analysis of pulmonary metastasectomy for hepatocellular carcinoma. Eur J Cardiothorac Surg 2012;41:376382.

21 Yoon YS, Kim HK, Kim J, Choi YS, Shim YM, Paik SW, Kim K: Long-term survival and prognostic factors after pulmonary metastasectomy in hepatocellular carcinoma. Ann Surg Oncol 2010;17:2795-2801.

22 Chen F, Sato K, Fujinaga T, Sonobe M, Shoji T, Sakai H, Miyahara R, Bando T, Okubo K, Hirata T, Date H: Pulmonary resection for metastases from hepatocellular carcinoma. World J Surg 2008;32:2213-2217.

23 Koide N, Kondo H, Suzuki K, Asamura H, Shimada K, Tsuchiya R: Surgical treatment of pulmonary metastasis from hepatocellular carcinoma. Hepatogastroenterology 2007;54:152-156.

24 Han KN, Kim YT, Yoon JH, Suh KS, Song JY, Kang CH, Sung SW, Kim JH: Role of surgical resection for pulmonary metastasis of hepatocellular carcinoma. Lung Cancer 2010;70:295-300.

25 Mizuguchi S, Nishiyama N, Izumi N, Tsukioka T, Komatsu H, Iwata T, Tanaka S, Takemura S, Kubo S: Clinical significance of multiple pulmonary metastasectomy for hepatocellular carcinoma. World J Surg 2016;40:380387.

26 Takahashi Y, Ikeda N, Nakajima J, Sawabata N, Chida M, Horio H, Okumura S, Kawamura M: Prognostic analysis of surgical resection for pulmonary metastasis from hepatocellular carcinoma. World J Surg 2016;40:21782185. 